\title{
ON THE EXISTENCE AND UNIQUENESS OF STREBEL DIFFERENTIALS
}

\author{
BY JOHN H. HUBBARD ${ }^{1,2}$ AND HOWARD MASUR ${ }^{1}$
}

Communicated August 5, 1975

Let $X$ be a compact Riemann surface of genus $g>1$, and $q \in H^{0}\left(X, \Omega^{\otimes_{2}}\right)$ be a holomorphic quadratic form on $X$. A tangent vector $\xi \in T_{x} X$ is called horizontal if $\langle q, \xi \otimes \xi\rangle>0$. The horizontal vectors define a foliation of $X$ singular at the zeroes of $q$. The form $q$ is called a Strebel form if the leaves of this foliation are compact.

If $q$ is a Strebel form, the leaves of the foliation through a zero of $q$ form a graph $\Gamma_{q}$, and $X-\Gamma_{q}$ is a union of metric straight cylinders (for the metric $\left.|q|^{1 / 2}\right)$. The central circles in each cylinder form a set of disjoint, nonpairwise homotopic and homotopically nontrivial simple closed curves on $X$, called the system of curves associated to $q$.

Let $M$ be an oriented differentiable compact surface of genus $g$, and $\mathrm{C} \mathrm{a}$ system of $n$ simple closed curves on $M$, disjoint, not pairwise homotopic and homotopically nontrivial. In the vector bundle $Q$ of pairs $(\theta, q)$, with $\theta$ in the Teichmüller space $\Theta_{M}$ (see [2] for notation) and $q$ a quadratic form on the Riemann surface above $\theta$, consider the space $E_{C} \subset Q$ of Strebel forms whose associated system of curves is homotopic to $C$. Denote $\pi: E_{C} \rightarrow \Theta_{M} \times \mathbf{R}_{+}^{n}$ the map whose first factor is the canonical projection, and whose second factor gives the heights of the cylinders. Our main result is the following

THEOREM. The map $\pi: E_{C} \rightarrow \Theta_{M} \times \mathbf{R}_{+}^{n}$ is a homeomorphism.

A similar result was proved by Strebel [4], with a very different proof. Further information can be found in [1], [4] and [5]. We wish to thank A. Douady for his most valuable help.

It is easy to check that a point $q$ in $E_{C}$ is completely determined by the homotopy class of its critical graph $\Gamma_{q}$ in $M$, the lengths of the segments of $\Gamma_{q}$, the heights of the cylinders and parameters measuring "the twisting around the central circles" of each cylinder. This allows an elementary, geometric and useful [3] description of Riemann surfaces.

The proof of the theorem proceeds in three steps: proving that $\pi$ is proper that $\pi$ is a local homeomorphism, and that $E_{C}$ is connected. The result then

AMS (MOS) subject classifications (1970). Primary 32G15.

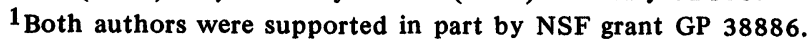

${ }^{2}$ First author was supported in part by Army Grant no. DA-ARO-D-31-124-73-G192. 
follows from the fact that $\Theta_{M}$ is simply connected. We shall give below an outline of the proof of each step; details will appear elsewhere.

\section{Proposition 1. The map $\pi$ is proper.}

Consider the union $E=\bigcup E_{C^{\prime}}$, where $C^{\prime}$ is a subset of $C$. Since integral curves of vector fields depend continuously on the vector field, $E$ is closed in $Q$. The map $\pi$ can be extended continuously to $E$ by assigning height zero to degenerate cylinders. The proposition then follows from the fact that the unit sphere bundle in $Q$ is proper over $\Theta_{M}$, and the following lemma:

LEMMA. If $\Gamma$ is a homotopy class of closed curves on $M$, the function $q \longmapsto \inf _{\gamma \in \Gamma} \int_{\gamma}|q|^{1 / 2}$ is continuous on $Q$.

The space $E_{k}$. Denote by $P_{k}$ the space of polynomial quadratic forms on $\mathrm{C}$ of the form $\left(z^{k}+p(z)\right) d z^{2}$, with $p$ a polynomial of degree at most $k-2$. It is easily checked that $P_{k}$ is a versal deformation of $z^{k} d z^{2}$ near $z=0$. Let $E_{k} \subset$ $P_{k}$ be the set of quadratic forms $q$ with connected critical graph $\Gamma_{q}$. For any $x \neq 0$ in $\mathbf{C}$ the function $f(q)=\lim \int_{x}^{\Gamma} q \sqrt{q}$ is well defined near $p=\backslash 0$ in $E_{k}$. Embed $E_{k}$ in $P_{k} \times \mathbf{R}$ by $q \longmapsto(q, f(q))$.

Proposition 2. The image of $E_{k}$ in $P_{k} \times \mathbf{R}$ is a differentiable submanifold of $P_{k} \times \mathbf{R}$ near 0 . The tangent space $T_{0} E_{k}$ is the set of pairs $(p, s)$ where; if $k$ is even, $p$ is a polynomial whose coefficients of degree $<k / 2$ vanish, and $s$ is arbitrary; and if $k$ is odd, the coefficients of degree $<(k-1) / 2$ vanish, and $s=1 / 2 \operatorname{Im} \int_{0}^{x} p / z^{k / 2} d z$.

The main step in the proof is to show that if $p(z)$ is tangent to $E_{k}$ at $q$, and $q$ has simple zeroes, then $p$ must have nonzero coefficients above the middle degree. On the Riemann surface of $\sqrt{q}$, forms $p / \sqrt{q}$ with $\operatorname{deg} p \leqslant[(k-3) / 2]$ form a basis for the holomorphic differentials, and the integrals of such forms over curves covering the bounded segments of $\Gamma_{q}$ cannot all be real. The result then follows from induction on $k$ and from the homogeneity of $E_{k}$.

The map $\pi$ is a local homeomorphism. Let $q_{0} \in E_{\mathrm{C}}$ vanish at points $x_{1}$, $\ldots, x_{m}$ to orders $k_{1}, \ldots, k_{m}$. Then a neighborhood $U$ of $q$ in $Q$ parametrizes deformations of the zeroes of $q_{0}$, and we get a map $U \rightarrow \Pi P_{k_{i}}$ classifying these deformations. The fibre product $V$ of $U$ and $\Pi E_{k_{i}}$ over $\Pi P_{k_{i}}$ is a differentiable manifold (but not a submanifold of $Q$ ), and parametrizes the deformations of $q$ "locally Strebel" near the zeroes of $q$. Because of the last coordinate in $E_{k}$, the functions $q \mapsto \operatorname{Im} \int_{\Gamma_{j}}^{\Gamma_{i}} \sqrt{q}$ are differentiable on $V$, where $\Gamma_{i}$ is the critical graph of $q$ near $x_{i}$, and the integral is over a path near a segment of $\Gamma q_{0}$. The equations $\operatorname{Im} \int_{\Gamma_{j}}^{\Gamma_{i}} \sqrt{q}=0$ over all such segments define $E$ as a submanifold of $Q \times$ $\mathbf{R}_{+}^{n}$, the last coordinate being heights. 
Proposition 3. The map $\pi$ is differentiable, and its derivative is an isomorphism.

The proof depends on a decomposition of $H^{1}\left(X, T_{X}\right)$ into those deformations leaving the zeroes of $q$ unchanged, and deformations with support in small neighborhoods of the $x_{i}$ 's.

Proposition 4. The space $E_{C}$ is connected.

The proof is by induction on the number of curves in $C$. It uses Lemma 1 and the following result [4]:

Lemma 2. Let $q$ be a Strebel differential on $X$, determining annuli $A_{1}$, $\ldots, A_{n}$ of moduli $M_{1}, \ldots, M_{n}$ and circumferences $a_{1}, \ldots, a_{n}$ (with respect to $\left.|q|^{1 / 2}\right)$. Let $B_{1}, \ldots, B_{n}$ be disjoint annuli on $X$, of moduli $N_{1}, \ldots, N_{n}$, and homotopic to $A_{1}, \ldots, A_{n}$ respectively. Then $\Sigma a_{i}^{2} M_{i} \geqslant \Sigma a_{i}^{2} N_{i}$, and equality occurs only if $A_{i}=B_{i}, i=1, \ldots, n$.

In case $C$ consists of a maximal system of $3 g-3$ curves, we are able to characterize the graph in terms of the heights and circumstances of the cylinders, and prove Proposition 4 directly.

\section{BIBLIOGRAPHY}

1. A. Douady and J. Hubbard, On the density of Strebel forms, Invent. Math. (to appear).

2. J. Hubbard, Sur les sections analytiques de la courbe universelle de Teichmüller, Mem. Amer. Math. Soc. (to appear).

3. H. Masur, On a class of geodesics in Teichmüller space, Ann. of Math. (to appear).

4. K. Strebel, Über quadratische Differentiale mit geschlossenen Trajektorien und extremale quasikonformen Abbildungen, Festband 70; Geburtstag von Rolf Nevanlinna, Springer, Berlin, 1966, pp. 105-127. MR 35 \#368.

5. - On quadratic differentials and extremal quasiconformal mappings, University of Minnesota Lecture notes, 1967.

DEPARTMENT OF MATHEMATICS, HARVARD UNIVERSITY, CAMBRIDGE, MASSACHUSETTS 02138 